\title{
Bimodal Porous Scaffolds by Sequential Electrospinning of Poly(glycolic acid) with Sucrose Particles
}

\author{
B. Wulkersdorfer, ${ }^{1}$ K. K. Kao, ${ }^{1,2}$ V. G. Agopian, ${ }^{1,2}$ A. Ahn, ${ }^{1}$ J. C. Dunn, ${ }^{2,3}$ \\ B. M. Wu, ${ }^{2,3}$ and M. Stelzner ${ }^{1,2}$ \\ ${ }^{1}$ Department of Surgery, VA Greater Los Angeles Health Care System, Los Angeles, CA 90073, USA \\ ${ }^{2}$ Department of Surgery, University of California at Los Angeles, Los Angeles, CA 90095, USA \\ ${ }^{3}$ Department of Bioengineering, University of California at Los Angeles, Los Angeles, CA 90095, USA
}

Correspondence should be addressed to M. Stelzner, matthias.stelzner@med.va.gov

Received 20 November 2009; Revised 20 January 2010; Accepted 20 January 2010

Academic Editor: Shanfeng Wang

Copyright (c) 2010 B. Wulkersdorfer et al. This is an open access article distributed under the Creative Commons Attribution License, which permits unrestricted use, distribution, and reproduction in any medium, provided the original work is properly cited.

\begin{abstract}
Electrospinning is a method to produce fine, biopolymer mesh with a three-dimensional architecture that mimics native extracellular matrix. Due to the small fiber diameter created in this process, conventional electrospun scaffolds have pore sizes smaller than the diameter of most cells. These scaffolds have limited application in tissue engineering due to poor cell penetration. We developed a hybrid electrospinning/particulate leaching technique to create scaffolds with increased porosity and improved cellular ingrowth. Poly(glycolic acid) (PGA) and a sucrose-ethanol suspension were electrospun in equal, alternating sequences at intervals of one, two, and ten minutes each. The scaffolds revealed fiber mesh with micropores of $10 \mu \mathrm{m}$ and uniformly distributed sucrose particles. Particulate leaching of sucrose from the one- or two-minute scaffolds revealed honeycomb structures with interconnected macropores between 50 and $250 \mu \mathrm{m}$. Sucrose leaching from the ten-minute scaffolds resulted in laminated structures with isolated macropores between 200 and $350 \mu \mathrm{m}$. Macropore size was directly proportional to the duration of the sucrose spinning interval. After 24 hours of cell culture, conventionally spun scaffolds demonstrated no cellular penetration. Conversely, the PGA/sucrose scaffolds demonstrated deep cellular penetration. This hybrid technique represents a novel method of generating electrospun scaffolds with interconnected pores suitable for cellular ingrowth.
\end{abstract}

\section{Introduction}

Tissue engineering aims to develop "biological substitutes that restore, maintain, or improve tissue or organ function[1]". A commonly used approach has involved seeding cells harvested from donor tissue onto a threedimensional matrix and ultimately implanting the device, such that the cells grow, organize, and function to augment or replace the damaged organ [2]. An ideal bioengineered matrix would be biocompatible and biodegradable yet have sufficient mechanical integrity to maintain its architecture until the seeded cells produced a new extracellular matrix (ECM) and replicate the donor organ [3].

Various biodegradable polymers have been used to create biomimetic scaffolds for tissue engineering, including poly(lactic acid) (PLA), poly(epsilon-caprolactone) (PCL), poly(lactic-co-glycolic acid) (PLGA), and poly(glycolic acid) (PGA) [4-7]. These poly(alpha-hydroxyesters) are commonly employed for clinical use in suture material and wound dressings, and have well known degradation characteristics. In vivo, these polyesters degrade by random hydrolysis and enzymatic action [8]. PGA and PLGA can be completely degraded from two to four weeks, whereas PCL and PLA may take up to 12 to 24 months for complete mass loss [9].

Porous scaffolds are particularly suited for use in tissue engineering because they have several properties that mimic the ECM, including a large surface area, pore size suitable for cellular ingrowth, and a three-dimensional environment conducive to promoting cell to cell contact [10]. Various techniques have been developed to fabricate porous scaffolds including solvent casting and particulate leaching [11], freeze 
drying [12], phase separation [13], solid free-form fabrication [14], foaming [15, 16], sintering [17], electroblowing [18], and electrospinning [19].

Electrospinning, or electrostatic spinning, was first developed in the early 1930's by Formhals; however, it was not until the 1960's that Taylor elucidated the fundamentals of the jet forming process, sparking a renewed interest in the larger scientific community [19]. It was found that by spinning a polymer solution under a large electric potential, electrostatic forces overcome the weaker forces of surface tension in the liquid, and create a fine charged jet [20]. The jet is then carried in the direction of the electric field, all the while thinning and elongating. Due to inherent instabilities of the jet motion, a dense nonwoven randomly oriented mesh can be collected on the counter electrode [10]. The typical setup is simple and inexpensive; it consists of a collector, a syringe pump containing a polymer solution, and a high voltage power supply [21].

The fibers generated by electrospinning typically range from $10 \mathrm{~nm}$ to $10 \mu \mathrm{m}$ in diameter, depending on the material, concentration, viscosity, electrical potential, working distance, and ambient conditions [9, 22, 23]. Eichhorn and Sampson have demonstrated that pore radius is proportional to fiber diameter [24]. Effective pore sizes of electrospun scaffolds would be anticipated to be between 0.5 and $5 \mu \mathrm{m}$. Kwon et al. have developed electrospun matrices with fiber diameters ranging from $0.3 \mu \mathrm{m}$ to $7 \mu \mathrm{m}$ and pore sizes of 0.2 to $30 \mu \mathrm{m}$ using various biodegradable copolyesters [25]. In electrospun meshes, fiber diameters from $10 \mathrm{~nm}$ to $10 \mu \mathrm{m}$ in diameter have been shown to promote cell attachment [22]. However, paradoxically the small pore sizes would restrict cellular infiltration and proliferation of cells which typically range from 5 to $20 \mu \mathrm{m}$ [26]. In order to generate a scaffold with both fine fibers and larger pore sizes, an alternative technique had to be developed.

The purpose of this study is to generate electrospun PGA scaffolds with increased porosity by combining electrospinning with particulate leaching. We modified the conventional electrospinning technique by alternately spinning a polymer solution with a sucrose suspension and subsequently leaching the sucrose crystals.

\section{Materials and Methods}

2.1. Electrospinning. For the electrospinning process, a $10 \%$ weight/volume solution of poly(glycolic acid) (PGA; Lakeshore Biomaterials; Birmingham, AL) was dissolved in 1,1,1,3,3,3-hexafluoro-2-propanol (HFP; Sigma-Aldrich (Fluka, Milwaukee, WI) and mixed on an orbital shaker for at least 12 hours. The solution was dispensed through an 18-gauge blunt needle (Integrated Dispensing Solutions Inc., Agoura Hills, CA) attached to a $10 \mathrm{~mL}$ plastic syringe (Becton Dickenson and Company, Franklin Lakes, NJ) with a syringe pump (Harvard Apparatus, Holliston, MA) at a flow rate of $1 \mathrm{~mL} / \mathrm{h}$. A $5 \mathrm{~mm}$ thick copper rod collector (McMaster Carr Co, Atlanta, GA) covered by copper foil (Lyon Industries Chicago Inc., South Elgin, IL) was placed at a distance of $8 \mathrm{~cm}$ from the needle tip and rotated at 1,200 rpm. Electrospinning was performed by applying an electrical field of $28 \mathrm{kV}$ from

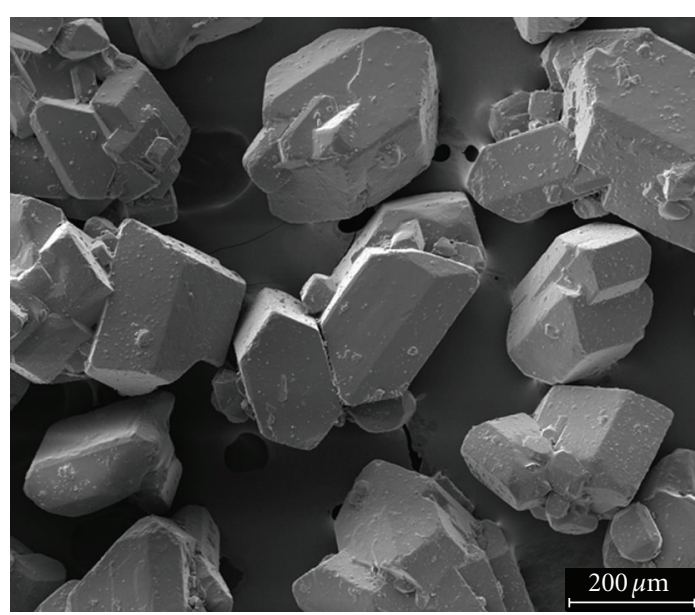

FIGURE 1: SEM image: sucrose particles.

a high voltage power source (Series PS/EL, Glassman High Voltage Inc., High Bridge, NJ) between the needle tip and the collector electrode. Nanofibers were collected on the target electrode rotating at $2000 \mathrm{rpm}$ to form a nonwoven tubular structure.

Confectioner's sugar (Pure Cane Sugar C\&H, C\&H Sugar Company, Inc., Crockett, CA) was sieved to a particle size ranging $250-350 \mu \mathrm{m}$ in diameter (Figure 1) and then a suspension of $70 \%$ weight/volume solution of sucrose with $70 \%$ ethanol (Fisher Scientific, Fairlawn, NJ) was prepared immediately prior to use.

Most sucrose crystals remained undissolved within the liquid phase, and the crystal suspension was of low viscosity. The sucrose-ethanol suspension was electrospun using the aforementioned apparatus at a distance of $15 \mathrm{~cm}$ to the same target collector used for the PGA.

To determine the effects of spin time, PGA and sucrose solutions were alternately spun in $1: 1$ ratios at time intervals of one, two, and ten minutes each (i.e., one minute of PGA spinning followed by one minute of sucrose spinning; two minutes of PGA spinning followed by two minutes of sucrose spinning; ten minutes of PGA spinning followed by ten minutes of sucrose spinning). Electrospinning using the PGA solution was alternated with the sucrose solution to create multi-layered scaffolds of 20 to 40 layers. PGA scaffolds spun without sucrose served as controls. After fabrication the electrospun tube was carefully removed from the target. The tube was leached twice in $37^{\circ} \mathrm{C}$ warm distilled water for ten minutes followed by dehydration in a hood under constant airflow.

2.2. Scanning Electron Microscopy (SEM). Before and after particulate leaching, $500 \mu \mathrm{m}$ cross sections of the electrospun tubes were prepared, sputter-coated with gold (Hummer II, Technics, Alexandria, VA) and imaged by scanning electron microscope (SEM) (Dual Beam NOVA 600, FEI Company, Portland, OR). Images were acquired at an accelerating voltage of $20 \mathrm{kV}$. For each experimental group, five images from different areas of the scaffolds were randomly picked and evaluated by a blinded observer to measure pore sizes. 


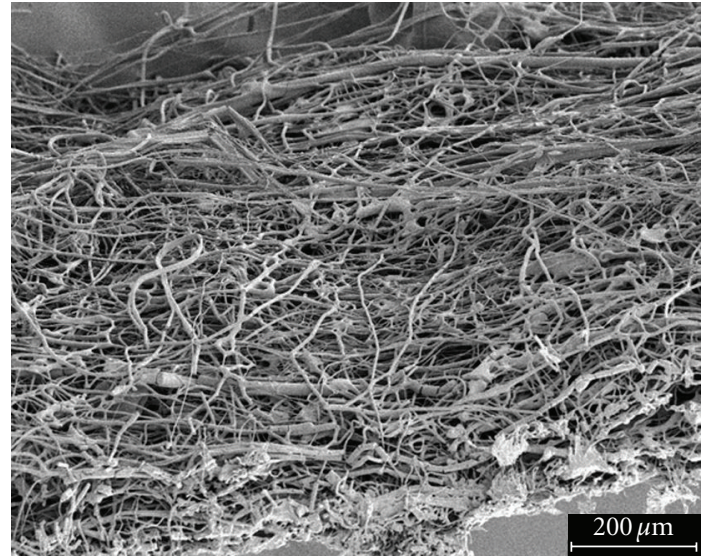

FIGURE 2: SEM image: cross-sectional view of control electrospun tube.

An average pore size was expressed as mean \pm standard error of mean.

2.3. Cell Culture. NIH/3T3 fibroblasts (ATCC, Manassas, VA) were cultured in cell-specific complete growth media (ATCC, Manassas, VA) supplemented with 10\% bovine calf serum (Colorado Serum Company, Denver, CO) and $1 \%$ antibiotic solution $(20,000 \mathrm{U} / \mathrm{mL}$ penicillin and $20,000 \mu \mathrm{g} / \mathrm{mL}$ streptomycin) (Cambrex BioScience, Walkersville, MD). This medium was used for all subsequent experimental steps. Cell cultures were maintained in a humidified $37^{\circ} \mathrm{C}$ cell culture incubator with a $95 \% \mathrm{O}_{2} / 5 \%$ $\mathrm{CO}_{2}$ environment. Culture media were replaced every three days. Fibroblasts were passaged three times, removed by $0.25 \%$ trypsin-EDTA treatment (Invitrogen-GIBCO, Carlsbad, CA), counted, and prepared for seeding onto the electrospun scaffolds.

2.4. Scaffold Preparation and Seeding. Electrospun tubes were cut into $1 \mathrm{~cm}^{2}$ sheets and subjected to plasma etching (Plasma Cleaner and Sterilizer, PDC-32G, Harrick Scientific, NY) for three minutes. The tubes were disinfected in $80 \%$ ethanol for 30 minutes, serially hydrated through an alcohol series in distilled water $(70 \%, 60 \%, 50 \%$ and $40 \%$ ethanol), subsequently rinsed with sterile phosphate buffered solution (PBS) (Fisher, Pittsburgh, PA) and dried under sterile conditions in a hood at room temperature for two hours. Prior to cell seeding, scaffolds were placed in a 24well cell culture plate and prewetted with culture medium. For each scaffold $2 \times 10^{5}$ fibroblasts were then suspended in $20 \mu \mathrm{L}$ complete growth media and placed as a drop in the center of the electrospun biopolymer mesh. To allow for cell attachment, the samples were incubated at $37^{\circ} \mathrm{C}$ for two hours before they were completely covered with media. Seeded scaffolds were harvested 24 hours after seeding. Scaffolds that received cell-free media served as negative controls.

2.5. Histological Evaluation. Samples were harvested and fixed in 10\% neutral buffered formalin (Fisher Diagnostics,
Middletown, VA), rinsed with PBS and embedded in OCT compound (Sakura Finetek, Torrance, CA). Embedded scaffolds were stored at $-80^{\circ} \mathrm{C}$ until sectioning. Cryosections were cut at $10 \mu \mathrm{m}$ thicknesses using a cryostat (Microm $\mathrm{GmbH}$, Walldorf, Germany) and stored at $-80^{\circ} \mathrm{C}$ until used. For fluorescent nuclear staining, frozen cryosections were taken from the $-80^{\circ} \mathrm{C}$ freezer and allowed to thaw and dry at room temperature for 15 minutes. Fluorescent staining was performed by adding mounting medium with $4^{\prime}$, 6-diamidino-2-phenylindole (DAPI) (Vector Laboratories, Burlingame, CA) to the sections and incubating them in a dark environment for 5 minutes at room temperature. DAPI-stained sections were evaluated under a fluorescent microscope (Image 1.42 software, National Institute of Health, http://rsbweb.nih.gov/ij/index.html; Carl Zeiss, Hallbergmoos, Germany) and evaluated at magnifications of $100 \times$ and $200 \times$. Five sections per experimental group taken from different areas of the cell seeded scaffolds were randomly picked and analyzed for cell penetration depth within the scaffolds. Due to variations in scaffold thickness, exact micron measurements of cell penetration were not useful; instead, a blinded observer was asked to assign subjective penetration scores based on perceived cell depth with the following scores: no penetration $(0)$, very poor penetration $(+)$, moderate penetration $(++)$, or deep penetration $(+++)$.

\section{Results}

Representative SEM samples of control and sequential electrospun scaffolds are shown in Figures 2-5. The control scaffold, that is, PGA without sucrose particles, revealed the typical architecture and random fiber distribution of a conventional electrospun mesh (Figure 2). Fiber diameters varied between $1-5 \mu \mathrm{m}$ and pores measured $10 \mu \mathrm{m}$ on average; no macropores were found.

Our maximum electrospinning intervals for PGA and sucrose were ten minutes. Preliminary experiments with 15minute spinning time intervals produced delaminated layers (data not shown). Minimum electrospinning intervals were one minute. Spinning intervals for PGA and sucrose less than one minute proved to be unreliable due to equipment limitations.

All sequentially electrospun scaffolds revealed randomly oriented PGA fibers with diameters between 1-5 $\mu \mathrm{m}$. The average macropore size ranged from $50-300 \mu \mathrm{m}$ with diameters correlating with spinning time intervals. Ten-minute intervals created the largest pores and one-minute intervals created the smallest pores (Figure 6).

Spinning for one minute resulted in macropore sizes ranging from 50 to $150 \mu \mathrm{m}$, spinning for two minutes created macropore sizes from 100 to $250 \mu \mathrm{m}$, and spinning for ten minutes revealed macropore sizes from 200 to $350 \mu \mathrm{m}$ in diameter. Pore sizes within areas of PGA mesh without sucrose averaged $10 \mu \mathrm{m}$ in diameter.

The density of sucrose distribution varied with time. With ten-minute sequential time intervals, the scaffolds revealed dense packing of incorporated sucrose crystals (Figure 3(a)). One- and two-minute alternating sequences 


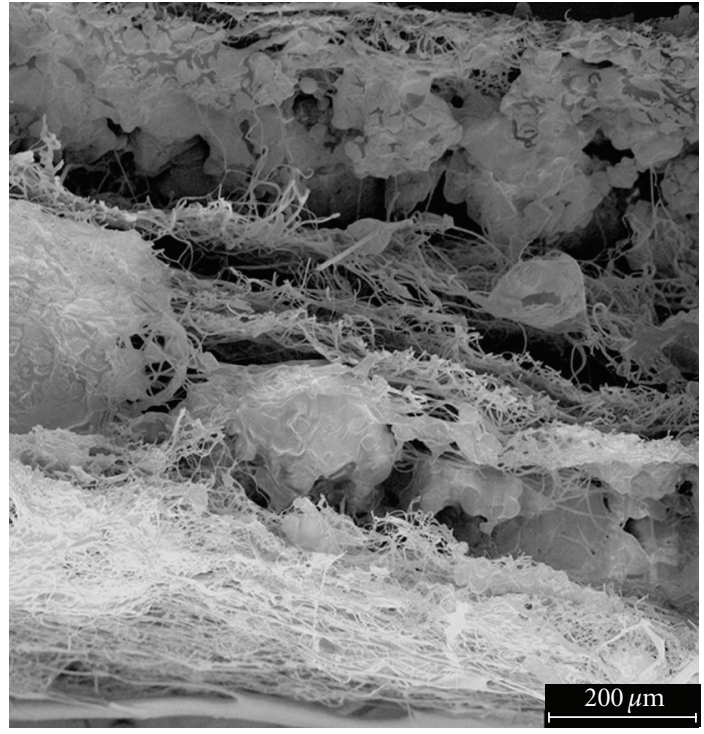

(a) Sugar particles embedded in the PGA scaffold

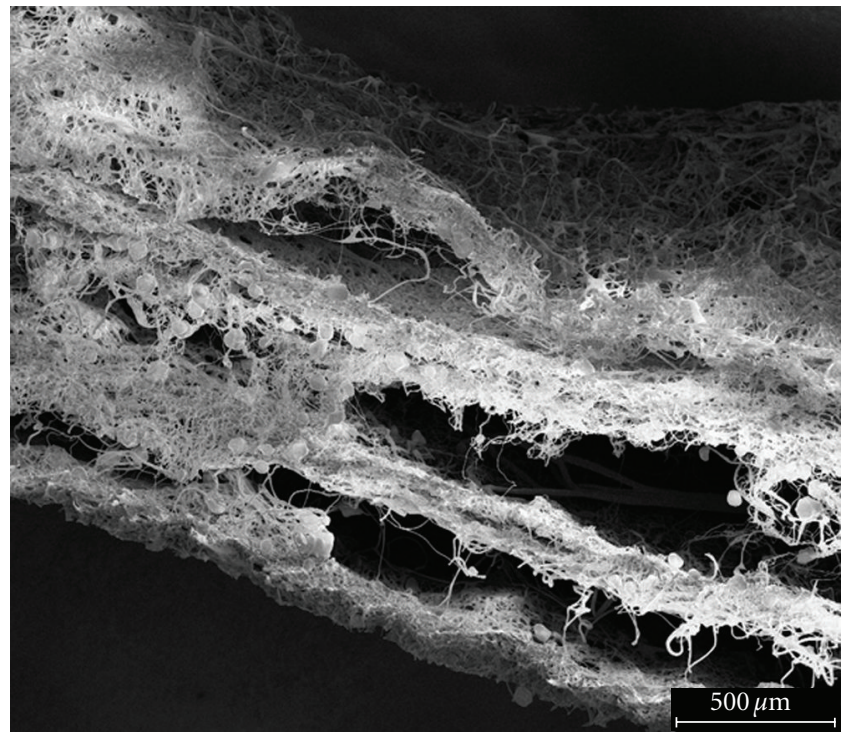

(b) Scaffold after leaching of sugar crystals

FIGURE 3: SEM images: cross-sectional view of electrospun tubes; the electrospinning interval was 10 minutes.

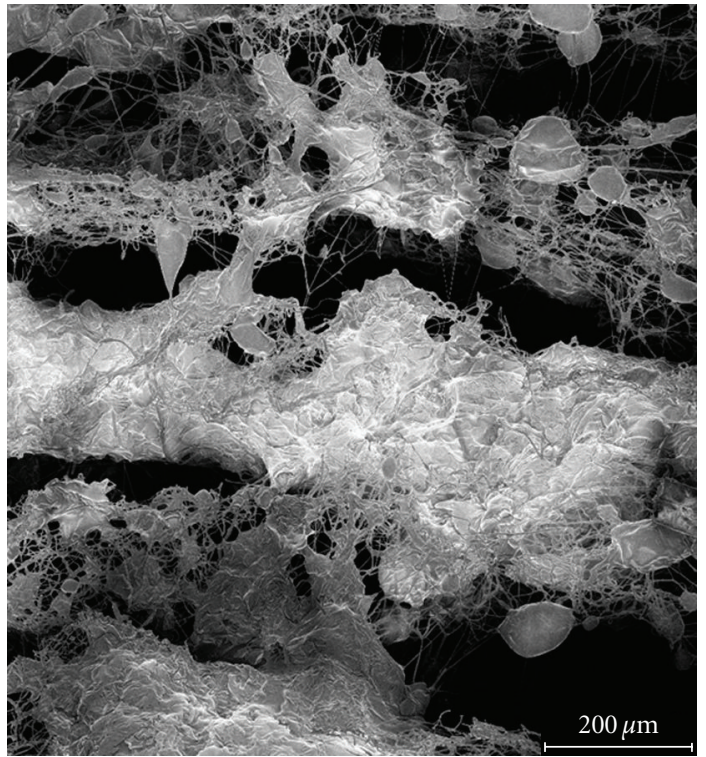

(a) Sugar particles embedded in the PGA scaffold

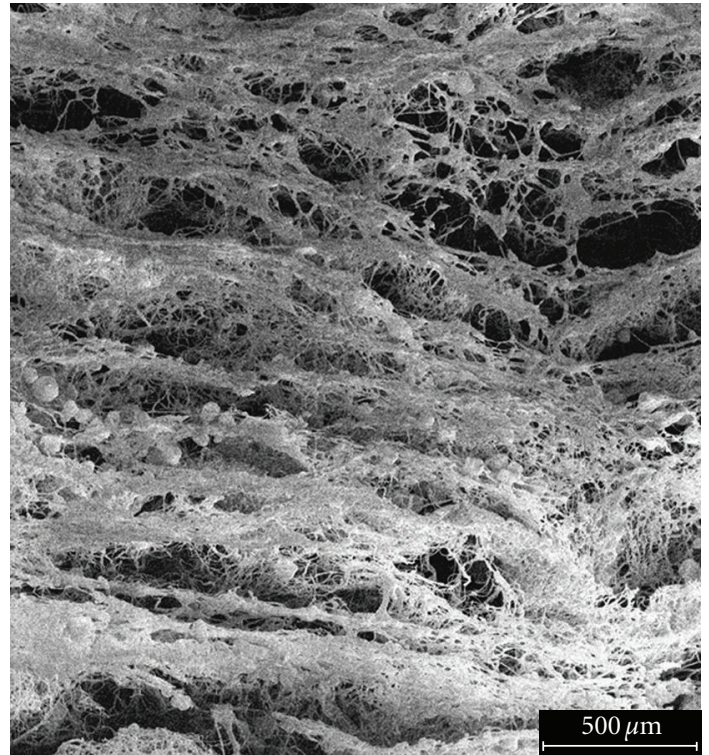

(b) Scaffold after leaching of sugar crystals

FIGURE 4: SEM images: cross-sectional view of electrospun tubes; the electrospinning interval was 2 minutes.

resulted in crystals that were less densely packed (Figures 4(a) and 5(a)). All samples demonstrated homogenous distribution of sucrose crystals.

PGA matrix architecture also demonstrated variability that correlated with interval times. Ten-minute time intervals produced thick, dense mesh layers of PGA fibers that were clearly discernible from the PGA-embedded sucrose layers (Figure 3(a)). In contrast, one- or two-minute intervals created a more integrated honeycomb-like structure of PGA and sucrose layers (Figures 4(a) and 5(a)). Two-minute intervals resulted in a coarser honeycomb structure, while the one-minute spinning time formed a more delicate network. Fine fibers of $2-5 \mu \mathrm{m}$ in diameter appeared suspended in the lumen of most pores.

Leaching of the scaffolds completely dissolved the embedded sucrose, leaving macroporous PGA structures in all cases (Figures 3(b), 4(b), and 5(b)). Sucrose "pockets" lined with PGA fibers may be seen. The tubular structures were form-stable and did not collapse. However, macropore sizes after leaching were reduced approximately $5-10 \%$. Of note, sucrose particles averaged 250 to $350 \mu \mathrm{m}$ (Figure 1), whereas average macropore diameter ranged between 50 to 


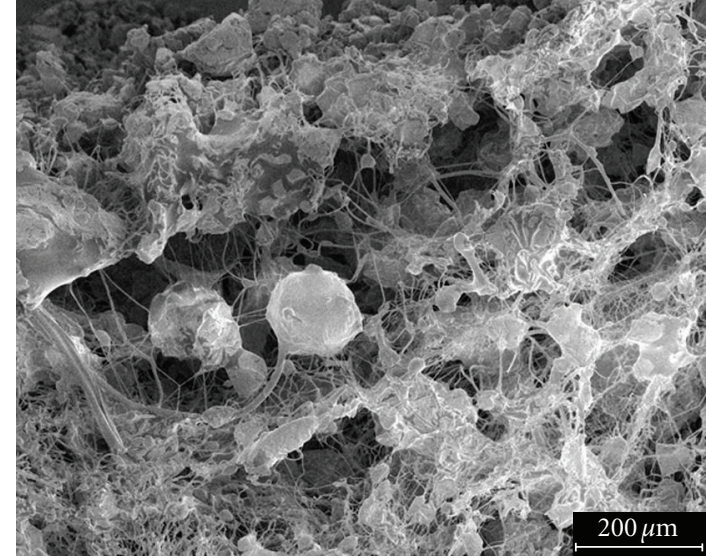

(a) Sugar particles embedded in the PGA scaffold

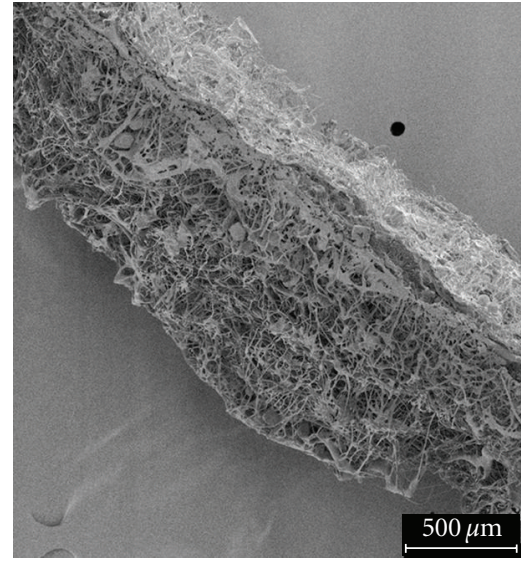

(b) Scaffold after leaching of sugar crystals

FIGURE 5: SEM images: Cross-sectional view of electrospun tubes; the electrospinning interval was 1 minute.
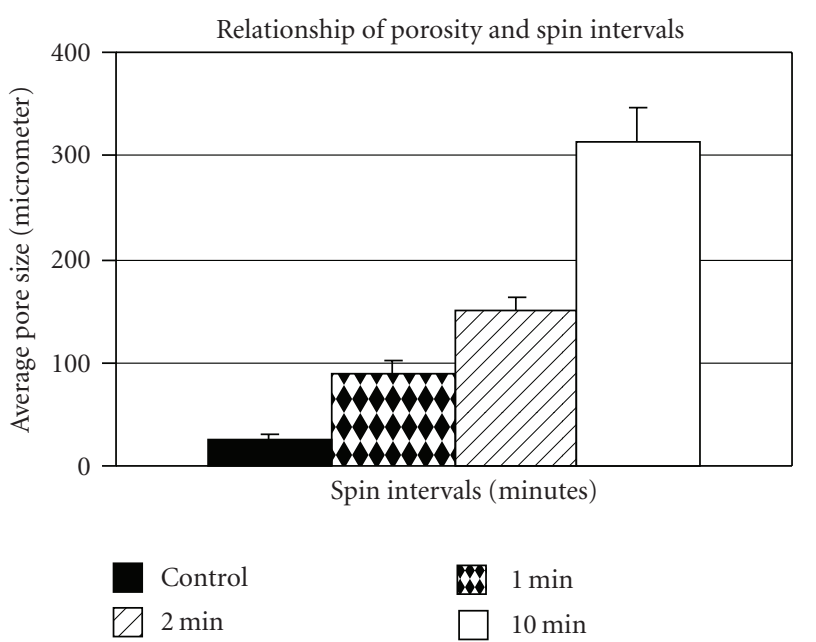

FIGURE 6: Relationship between porosity and spin intervals.

$350 \mu \mathrm{m}$. Ten-minute intervals left alternating layers of void space and dense PGA mesh layers. Rare communication between macropores and periodic layer delamination was noted in the ten-minute interval scaffolds (Figure 3(b)). One- and two-minute time intervals revealed honeycomblike structures after sucrose leaching. The PGA fiber pockets were seen to communicate through defects in the PGA mesh layers, thus creating macroporous interconnections (Figures 4 (b) and $5(\mathrm{~b})$ ).

Interconnection of macropores in the one- and twominute spun scaffolds was confirmed by our cell penetration assay (Figure 7).

After cell seeding, fibroblasts attached to the interior of the material. Nuclear staining with DAPI indicated that seeded cells were located on and within the electrospun scaffolds. Penetration into the deeper layers of the mesh was variable. Despite exhibiting larger macropores, tenminute interval scaffolds (Figures $7(\mathrm{c})$ and $7(\mathrm{~d})$ ) and control scaffolds (Figures 7(a) and 7(b)) had cells attached to the
TABLE 1: Penetration scores for five randomly selected sections from each study group.

\begin{tabular}{lcccc}
\hline & Control & 10 minutes & 2 minutes & 1 minute \\
\hline Section 1 & 0 & + & ++ & ++ \\
Section 2 & + & + & ++ & ++ \\
Section 3 & 0 & ++ & ++ & + \\
Section 4 & + & + & + & +++ \\
Section 5 & O & + & +++ & + \\
\hline
\end{tabular}

( 0 denotes no penetration; +++ denotes deep penetration).

surface with only minimal cell penetration into the matrix. Although the ten-minute scaffolds showed slightly more cell penetration than the control matrix, the denser PGA fibers surrounding the large macropores appeared to create a barrier that prevented the cells from reaching the deeper structures of the scaffold. More pronounced cell penetration occurred in one-minute scaffolds. The deepest penetration (to about $250 \mu \mathrm{m}$ ) was found in two-minute scaffolds (Figures 7(e) and 7(f)). In all samples with cell penetration, cells within the scaffolds appeared to be distributed along the fibers lining the "walls" of the pores. Data representing a synopsis of these results are shown in Table 1.

\section{Discussion}

The combination of electrospinning sucrose particles and poly(glycolic acid) represents a hybrid method that incorporates particulate leaching to create a nonwoven scaffold with uniform fiber diameter, macropores, and interconnected pores of fixed size. With the traditional method of simple electrospinning, porosity is limited due to high scaffold density resulting from small fiber diameters. Cells seeded onto control samples without sucrose were unable to penetrate the scaffold surface and were not seen within the scaffold interior; the high fiber density and smaller pore sizes $(10 \mu \mathrm{m})$ inhibited cell penetration. In contrast, with our hybrid technique of electrospinning and particulate leaching 


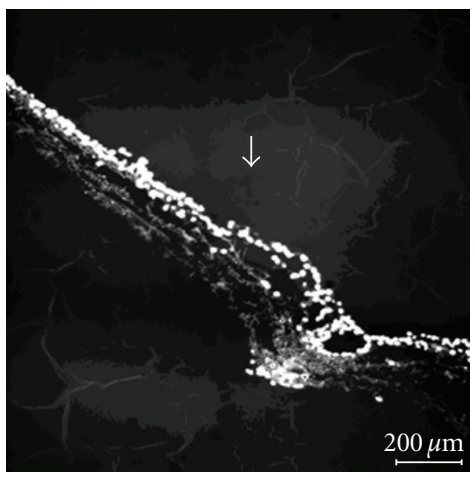

(a) Control scaffold: $(100 \times)$

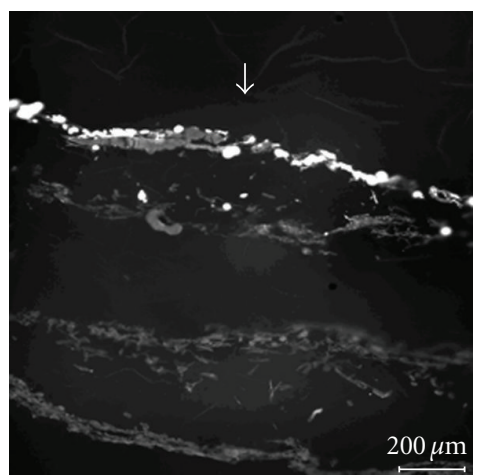

(c) Ten-minute scaffold: $(100 \times)$

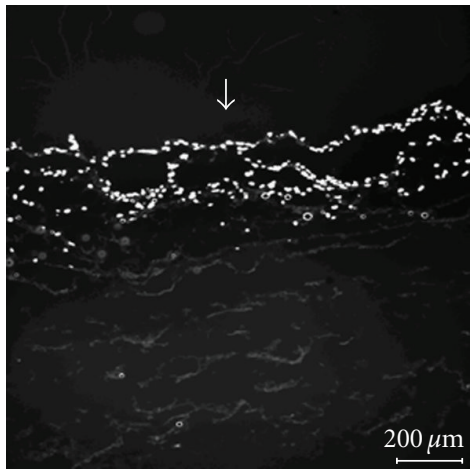

(e) Two-minute scaffold: $(100 \times)$

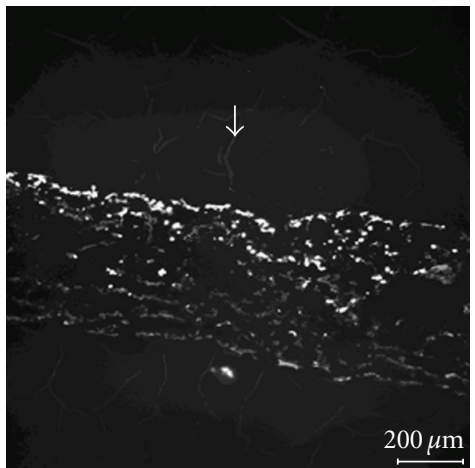

(g) One-minute scaffold: $(100 \times)$

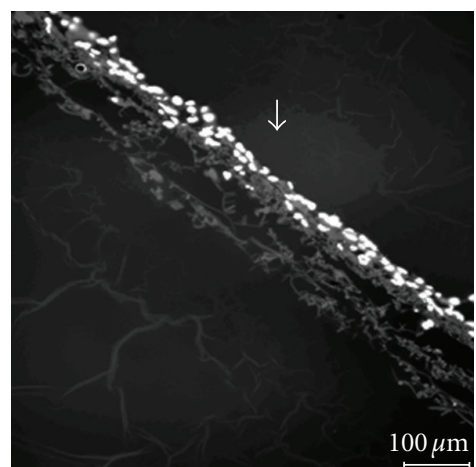

(b) Control scaffold: $(200 \times)$

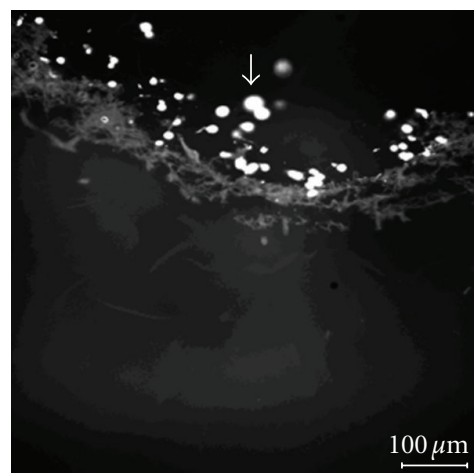

(d) Ten-minute scaffold: $(200 \times)$

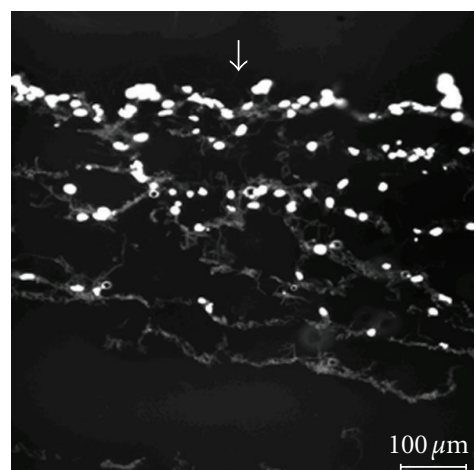

(f) Two-minute scaffold: $(200 \times)$

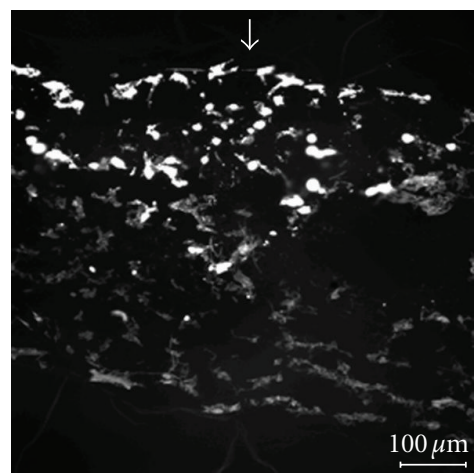

(h) One-minute scaffold: $(200 \times)$

Figure 7: Cryosections of seeded scaffolds with DAPI nuclear stained cells at 24 hours. White arrows point towards the scaffold seeding surface. 
we were able to fabricate a matrix with fiber diameters of $1-$ $5 \mu \mathrm{m}$ and a larger pore structure in the range of $50-350 \mu \mathrm{m}$. In addition, we were able to control the pore distribution and interconnectedness by varying the duration of both sucrose-ethanol and polymer spinning times. The result was a homogenous distribution of pores throughout the architecture that was unaffected by the evaporation of the solvents.

Macropore size was dictated by porogen diameter and spinning times. Though the average size of sucrose crystals was $250-350 \mu \mathrm{m}$, pore sizes as small as $50 \mu \mathrm{m}$ were found. This was likely due to the sifting process which included smaller sucrose crystals within the sucrose-ethanol solution. In future studies, ultrasonic size sorting could be employed to more accurately separate out porogens of a desired size.

Spinning time was the fundamental variable in the creation of macroporous scaffolds. The upper and lower time limits of electrospinning during which the production of a form-stable, porous scaffolds may be fabricated were one and ten minutes, respectively. With shorter electrospinning times of one or two minutes, scaffolds suitable for cell infiltration and migration were generated with apparent honeycomblike structures and interconnected pores of $50-250 \mu \mathrm{m}$. Conversely, with an electrospinning time interval of ten minutes, macroporous layers created by sucrose deposition alternated with denser layers of PGA fibers. Though the pore size was larger $(200$ to $350 \mu \mathrm{m})$ in these scaffolds due to increased sucrose deposition, the ten-minute time interval resulted in a laminated structure with decreased pore interconnection which limited cellular infiltration. In the manufacture of porous scaffolds, polymer and sucrose spinning times may be varied to determine macropore size and pore interconnectedness and to improve cellular penetration. In order to avoid laminated structures, future studies could involve co-spinning of the solutions using a double-spinneret technique. By cospinning PGA and sucrose solutions, a more uniform incorporation of sucrose particles within the fibers could potentially be obtained.

Other efforts to expand and control the pore sizes of electrospun scaffolds have been previously described [2628]. Kidoaki et al. introduced a new multilayer electrospinning technique to create ordered structures of type I collagen, styrenated gelatin, and segmented polyurethane. By sequentially spinning different polymers, the authors engineered more complex, hierarchically designed scaffolds than could be made by simple electrospinning alone. By mixing PLLA and polyethylene glycol, Pantojas et al. could modify fiber morphology such that nanometric pores were created [29]. Zhu et al. utilized a novel rotating frame cylinder as a collection device, which resulted in varying porosity based on rotational speed [30]. Nam et al. introduced sodium chloride crystals with poly(E-caprolactone) (PCL) in the electrospinning process [26]. During fabrication, salt particles were deposited by gravity onto the electrospun PCL fibers. This resulted in a compact structure with multiple layers. The PCL scaffold structure described by these authors resembled our ten-minute interval sample, with dense polymer fiber layers alternating with void space. However, in contrast to our technique, their PCL scaffolds lacked a homogenous porous architecture. This difference may be attributable to our use of sucrose suspended in solution rather than the direct use of a dry crystalline powder.

Leong et al. utilized a cryogenic technique to incorporate ice crystals into a poly(D,L-lactide) electrospun scaffold [28]. By varying the humidity of the electrospinning environment, they attained pore sizes ranging from $900-5000 \mu \mathrm{m}^{2}$. However, they found cell infiltration into the scaffolds to be limited to $50 \mu \mathrm{m}$. In our study cell penetration was variable; however, cells were found up to $250 \mu \mathrm{m}$ deep in the scaffold structure. This finding was in contrast to the results of Nam et al. As a consequence of using PCL, a polymer with significantly slower degradation kinetics, Nam's group could seed and culture cells on scaffolds for up to 3 weeks. This was significantly longer than our 24-hour incubation period. Consequently, they observed cell penetration and proliferation deep within the scaffold at 3000 to $6000 \mu \mathrm{m}$.

Though we successfully generated highly porous electrospun scaffolds, our study had several limitations. There is not a definitive explanation for the correlation between electrospinning time and pore size. We demonstrated that pore sizes ranged from 50 to $150 \mu \mathrm{m}, 100$ to $250 \mu \mathrm{m}$, and 200 to $350 \mu \mathrm{m}$ in diameter with increasing spinning times. Most likely, increased sucrose deposition occurred with longer time intervals. In order to generate larger pores, crystal deposition would necessarily occur in areas immediately adjacent to one another, yet jet instability within the Taylor cone would dictate a more random distribution. Regardless, it has been noted that deposition of particles larger than $150 \mu \mathrm{m}$ may result in clustering [26]. As we utilized sucrose crystals $250-300 \mu \mathrm{m}$ in diameter, it is likely that clustered deposition occurred. Additional investigation utilizing different particle sizes is warranted to elucidate the relationship between electrospinning time, particle deposition, and pore size.

PGA is a linear aliphatic polyester with a high degree of crystallization (45-55\%) which renders it insoluble in most organic solvents; exposure to ethanol during scaffold production does not cause any structural changes [31]. In addition, wetting during cellular seeding does not induce any scaffold shrinkage. However, as may be seen in our SEM images (Figures 2(a), 3(a), and 4(a)) the sucrose-laden scaffolds appear to have several areas of "fused" PGA fibers or a "beads-on-a-string" appearance. This architecture may be the result of using a PGA solution with insufficient viscosity. As Kumbar et al. have demonstrated, decreasing polymer concentration, which implies decreased viscosity, may result in breaking down of the jet formed in the electrospinning process. The result would be an electrospray of microdroplets rather than fibers [32]. Though the areas of beading were minimal, future studies warrant investigation into increased concentrations of PGA solutions.

Another limitation of our study lay in our histologic assessment of cell penetration. Our method of assigning penetration scores was necessarily subjective, without absolute depth criteria. This was a consequence of the heterogeneity of scaffold thickness, and we felt that absolute measurements would have been misleading. In addition, our method of 
gravity-seeding may have limited cell adhesion and subsequent proliferation. Additional investigations of cell behavior on and in our scaffolds may be performed using new seeding methods, such as agitation [33], dynamic mixing [34], perfusion flow-seeding [35], or filtration seeding [36].

In our study, we investigated three electrospinning conditions to create porous scaffolds: (a) one minute of PGA spinning followed by one minute of sucrose spinning, (b) two minutes of PGA spinning followed by two minutes of sucrose spinning, and (c) ten minutes of PGA spinning followed by ten minutes of sucrose spinning. In each case, time intervals were kept constant throughout the entire sequence, with a 1:1 ratio of PGA and sugar spinning times. Further studies that focus on varying PGA or sugar solution spin times could be investigated. As discussed above, the ten-minute interval resulted in larger macropores, but reduced interconnectedness. Thus in order to increase pore size as well as interconnectedness, the ratio of PGA to sugar spinning times could be extended to a ratio of $1: 2$ or $1: 3$ in order to create a more honeycomb-like structure rather than a laminated structure.

\section{Conclusion}

We have identified a simple technique of sequential, alternating electrospinning of PGA and sucrose solutions. This method results in fine PGA fiber scaffolds with pores of $50-350 \mu \mathrm{m}$. Pore size can be controlled by varying the time intervals for each spinning sequence. Combination electrospinning represents an excellent modality to generate designer scaffolds with controllable pore sizes that promote cell attachment and penetration. Further studies will be needed to further refine this method.

\section{Acknowledgments}

The authors express their deepest thanks to Sergey V. Prikhodko, PhD, their SEM specialist, for his superb technical help as well as to $\mathrm{Xu}$ Yuhuan, $\mathrm{PhD}$, and Min Lee, $\mathrm{PhD}$, for their expert advice on electrospinning methods.

\section{References}

[1] R. Langer and J. P. Vacanti, “Tissue engineering," Science, vol. 260, no. 5110, pp. 920-926, 1993.

[2] T. C. Grikscheit and J. P. Vacanti, "The history and current status of tissue engineering: the future of pediatric surgery," Journal of Pediatric Surgery, vol. 37, no. 3, pp. 277-288, 2002.

[3] D. W. Hutmacher, "Scaffold design and fabrication technologies for engineering tissues - state of the art and future perspectives," Journal of Biomaterials Science, Polymer Edition, vol. 12, no. 1, pp. 107-124, 2001.

[4] B. Sharma and J. H. Elisseeff, "Engineering structurally organized cartilage and bone tissues," Annals of Biomedical Engineering, vol. 32, no. 1, pp. 148-159, 2004.

[5] F. Rosso, G. Marino, A. Giordano, M. Barbarisi, D. Parmeggiani, and A. Barbarisi, "Smart materials as scaffolds for tissue engineering," Journal of Cellular Physiology, vol. 203, no. 3, pp. 465-470, 2005.
[6] W. J. Li, K. G. Danielson, P. G. Alexander, and R. S. Tuan, "Biological response of chondrocytes cultured in threedimensional nanofibrous poly(epsilon-caprolactone) scaffolds," Journal of Biomedical Materials Research A, vol. 67, no. 4, pp. 1105-1114, 2003.

[7] W. J. Li, C. T. Laurencin, E. J. Caterson, R. S. Tuan, and F. K. Ko, "Electrospun nanofibrous structure: a novel scaffold for tissue engineering," Journal of Biomedical Materials Research, vol. 60, no. 4, pp. 613-621, 2002.

[8] N. Ashammakhi and P. Rokkanen, "Absorbable polyglycolide devices in trauma and bone surgery," Biomaterials, vol. 18, no. 1, pp. 3-9, 1997.

[9] D. Liang, B. S. Hsiao, and B. Chu, "Functional electrospun nanofibrous scaffolds for biomedical applications," Advanced Drug Delivery Reviews, vol. 59, no. 14, pp. 1392-1412, 2007.

[10] S. Y. Chew, Y. Wen, Y. Dzenis, and K. W. Leong, "The role of electrospinning in the emerging field of nanomedicine," Current Pharmaceutical Design, vol. 12, no. 36, pp. 4751-4770, 2006.

[11] R. C. Thomson, M. J. Yaszemski, J. M. Powers, and A. G. Mikos, "Hydroxyapatite fiber reinforced $\operatorname{poly}(\alpha$-hydroxy ester) foams for bone regeneration," Biomaterials, vol. 19, no. 21, pp. 1935-1943, 1998.

[12] P. X. Ma and R. Zhang, "Synthetic nano-scale fibrous extracellular matrix," Journal of Biomedical Materials Research, vol. 46, no. 1, pp. 60-72, 1999.

[13] R. Zhang and P. X. Ma, "Poly $(\alpha$-hydroxyl acids)/hydroxyapatite porous composites for bone- tissue engineering. I. Preparation and morphology," Journal of Biomedical Materials Research, vol. 44, no. 4, pp. 446-455, 1999.

[14] D. W. Hutmacher, "Scaffolds in tissue engineering bone and cartilage," Biomaterials, vol. 21, no. 24, pp. 2529-2543, 2000.

[15] M. H. Sheridan, L. D. Shea, M. C. Peters, and D. J. Mooney, "Bioabsorbable polymer scaffolds for tissue engineering capable of sustained growth factor delivery," Journal of Controlled Release, vol. 64, no. 1-3, pp. 91-102, 2000.

[16] L. Singh, V. Kumar, and B. D. Ratner, "Generation of porous microcellular 85/15 poly (DL-lactide-co-glycolide) foams for biomedical applications," Biomaterials, vol. 25, no. 13, pp. 2611-2617, 2004.

[17] Y. Kuboki, Q. Jin, and H. Takita, "Geometry of carriers controlling phenotypic expression in BMP-induced osteogenesis and chondrogenesis," Journal of Bone and Joint Surgery. America, vol. 83, supplement 1, part 2, pp. S105-115, 2001.

[18] I. C. Um, D. Fang, B. S. Hsiao, A. Okamoto, and B. Chu, "Electro-spinning and electro-blowing of hyaluronic acid," Biomacromolecules, vol. 5, no. 4, pp. 1428-1436, 2004.

[19] G. I. Taylor, "Electrically driven jets," Proceedings of the Royal Society of London, vol. 313, pp. 453-475, 1969.

[20] C. P. Barnes, S. A. Sell, E. D. Boland, D. G. Simpson, and G. L. Bowlin, "Nanofiber technology: designing the next generation of tissue engineering scaffolds," Advanced Drug Delivery Reviews, vol. 59, no. 14, pp. 1413-1433, 2007.

[21] Q. P. Pham, U. Sharma, and A. G. Mikos, "Electrospinning of polymeric nanofibers for tissue engineering applications: a review," Tissue Engineering, vol. 12, no. 5, pp. 1197-1211, 2006.

[22] S. V. Fridrikh, J. H. Yu, M. P. Brenner, and G. C. Rutledge, "Controlling the fiber diameter during electrospinning," Physical Review Letters, vol. 90, no. 14, Article ID 144502, 4 pages, 2003.

[23] S. G. Kumbar, R. James, S. P. Nukavarapu, and C. T. Laurencin, "Electrospun nanofiber scaffolds: engineering soft tissues," Biomedical Materials, vol. 3, no. 3, Article ID 034002, 2008. 
[24] S. J. Eichhorn and W. W. Sampson, "Statistical geometry of pores and statistics of porous nanofibrous assemblies," Journal of the Royal Society Interface, vol. 2, no. 4, pp. 309-318, 2005.

[25] I. K. Kwon, S. Kidoaki, and T. Matsuda, "Electrospun nano- to microfiber fabrics made of biodegradable copolyesters: structural characteristics, mechanical properties and cell adhesion potential," Biomaterials, vol. 26, no. 18, pp. 3929-3939, 2005.

[26] J. Nam, Y. Huang, S. Agarwal, and J. Lannutti, "Improved cellular infiltration in electrospun fiber via engineered porosity," Tissue Engineering, vol. 13, no. 9, pp. 2249-2257, 2007.

[27] S. Kidoaki, I. K. Kwon, and T. Matsuda, "Mesoscopic spatial designs of nano- and microfiber meshes for tissue-engineering matrix and scaffold based on newly devised multilayering and mixing electrospinning techniques," Biomaterials, vol. 26, no. 1, pp. 37-46, 2005.

[28] M. F. Leong, M. Z. Rasheed, T. C. Lim, and K. S. Chian, "In vitro cell infiltration and in vivo cell infiltration and vascularization in a fibrous, highly porous poly(D,L-lactide) scaffold fabricated by cryogenic electrospinning technique," Journal of Biomedical Materials Research, vol. 91, no. 1, pp. 231-240, 2008.

[29] V. M. Pantojas, E. Velez, D. Hernandez, and W. Otano, "Initial study on fibers and coatings for the fabrication of bioscaffolds," Puerto Rico Health Sciences Journal, vol. 28, no. 3, pp. 258-265, 2009.

[30] X. Zhu, W. Cui, X. Li, and Y. Jin, "Electrospun fibrous mats with high porosity as potential scaffolds for skin tissue engineering," Biomacromolecules, vol. 9, no. 7, pp. 1795-1801, 2008.

[31] J. C. Middleton and A. J. Tipton, "Synthetic biodegradable polymers as orthopedic devices," Biomaterials, vol. 21, no. 23, pp. 2335-2346, 2000.

[32] S. G. Kumbar, S. Bhattacharyya, S. Sethuraman, and C. T. Laurencin, "A preliminary report on a novel electrospray technique for nanoparticle based biomedical implants coating: precision electrospraying," Journal of Biomedical Materials Research B, vol. 81, no. 1, pp. 91-103, 2007.

[33] K. J. L. Burg, W. D. Holder Jr., C. R. Culberson, et al., "Comparative study of seeding methods for three-dimensional polymeric scaffolds," Journal of Biomedical Materials Research, vol. 51, no. 4, pp. 642-649, 2000.

[34] G. Vunjak-Novakovic, B. Obradovic, I. Martin, P. M. Bursac, R. Langer, and L. E. Freed, "Dynamic cell seeding of polymer scaffolds for cartilage tissue engineering," Biotechnology Progress, vol. 14, no. 2, pp. 193-202, 1998.

[35] S. S. Kim, C. A. Sundback, S. Kaihara, et al., "Dynamic seeding and in vitro culture of hepatocytes in a flow perfusion system," Tissue Engineering, vol. 6, no. 1, pp. 39-44, 2000.

[36] Y. Li, T. Ma, D. A. Kniss, L. C. Lasky, and S. T. Yang, "Effects of filtration seeding on cell density, spatial distribution, and proliferation in nonwoven fibrous matrices," Biotechnology Progress, vol. 17, no. 5, pp. 935-944, 2001. 

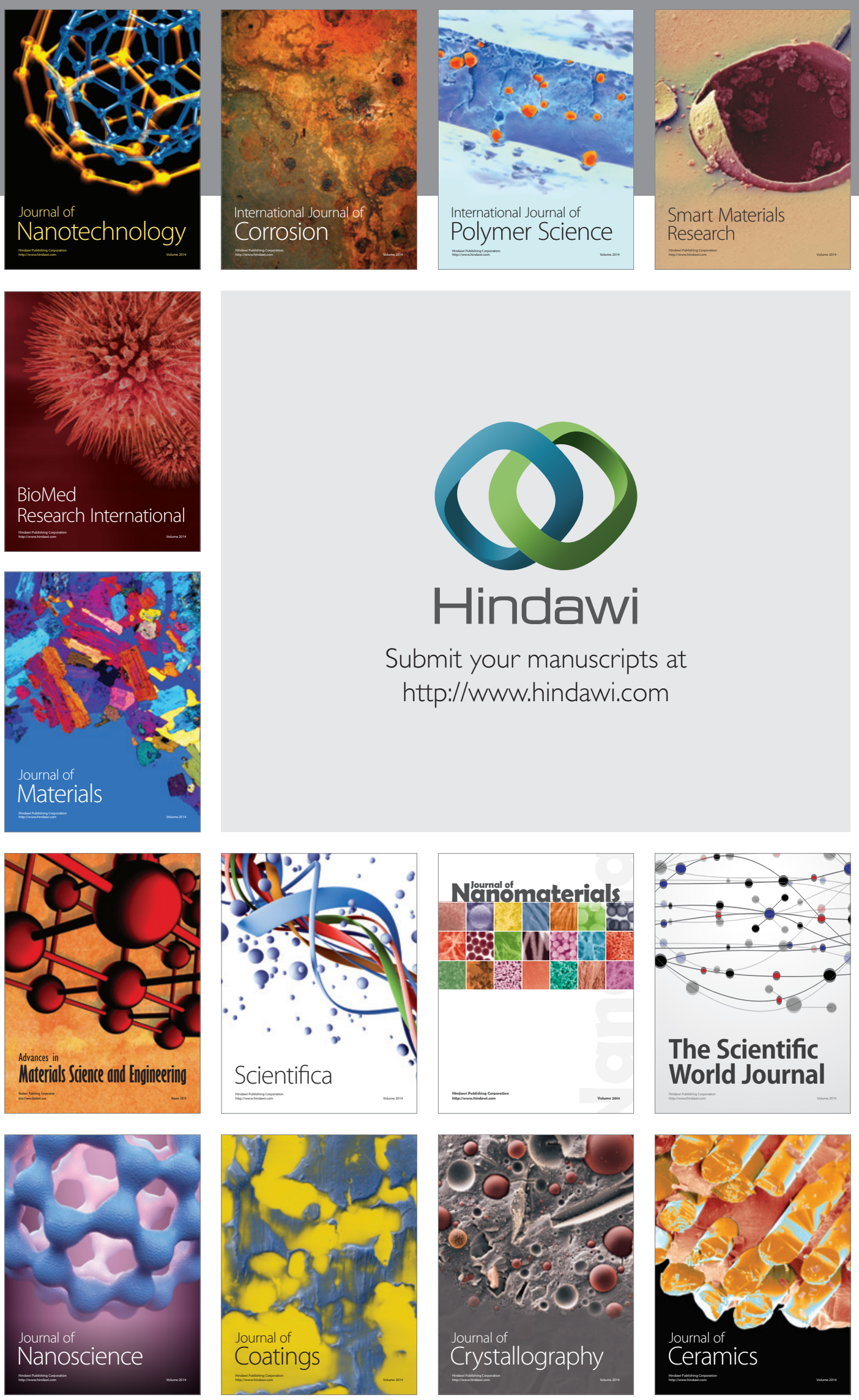

The Scientific World Journal

Submit your manuscripts at

http://www.hindawi.com

\section{World Journal}

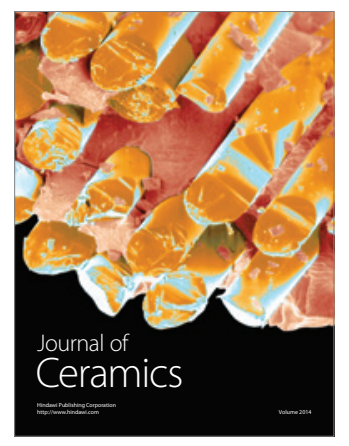

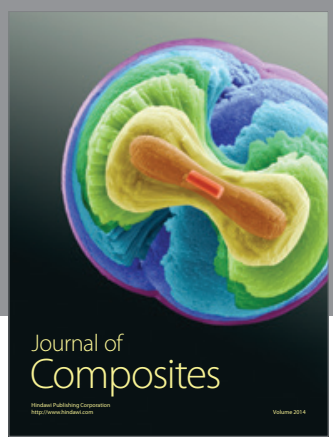
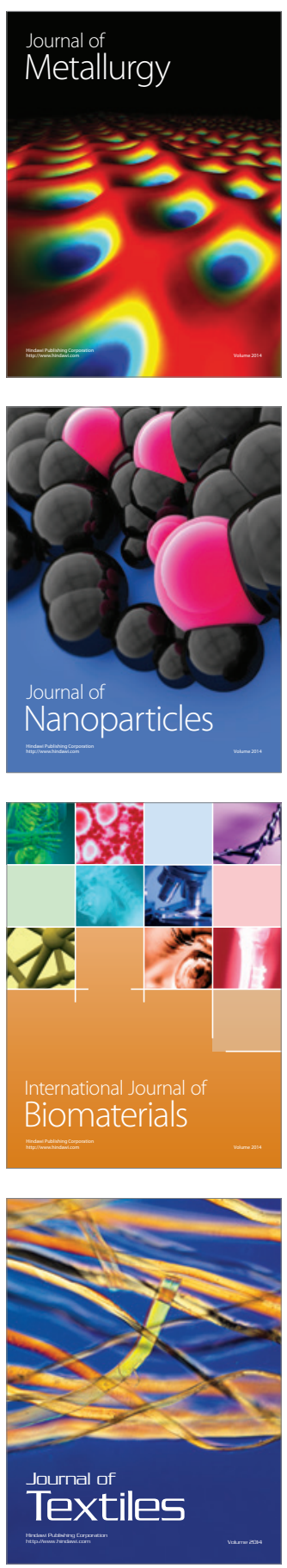